\section{Medíąl Seienee}

pISSN 2321-7359; eISSN 2321-7367

To Cite:

Giri A, Acharya S, Andhale A, Kumar S, Singh RK, Chandankhede A. Neurofibromatosis type - 1 (NF-1) presenting as intracerebral

haemorrhage in a young adult- unveiling a rare culprit. Medical Science, 2022, 26, ms52e2019.

doi: https://doi.org/10.54905/disssi/v26i120/ms52e2019

\section{Author Affiliation:}

${ }^{1}$ Resident, Dept. of Medicine, Jawarharlal Nehru Medical College and Acharya Vinoba Bhave, Sawangi Meghe, Wardha, Maharashtra, India ${ }^{2}$ Professor and Head, Dept. of Medicine, Jawarharlal Nehru Medical College and Acharya Vinoba Bhave, Sawangi Meghe, Wardha,

Maharashtra, India

${ }^{3}$ Senior Resident, Dept. of Medicine, Jawarharlal Nehru Medical College and Acharya Vinoba Bhave, Sawangi Meghe, Wardha, Maharashtra, India

${ }^{4}$ Professor, Dept. of Medicine, Jawarharlal Nehru Medical College and Acharya Vinoba Bhave, Sawangi Meghe, Wardha, Maharashtra, India ${ }^{5}$ Resident, Dept. of Radiodiagnosis, Jawarharlal Nehru Medical College and Acharya Vinoba Bhave, Sawangi Meghe, Wardha, Maharashtra, India

${ }^{6}$ Asst. Professor, Dept. Of Neurosurgery, Jawarharlal Nehru Medical College and Acharya Vinoba Bhave, Sawangi Meghe, Wardha, Maharashtra, India

Peer-Review History

Received: 05 December 2021

Reviewed \& Revised: 07/December/2021 to 24/January/2022

Accepted: 26 January 2022

Published: 30 January 2022

\section{Peer-review Method}

External peer-review was done through double-blind method.

URL: https://www.discoveryjournals.org/medicalscience

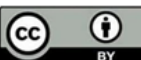

This work is licensed under a Creative Commons Attribution 4.0 International License.
Neurofibromatosis type -1

(NF-1) presenting as

intracerebral haemorrhage in a young adult- unveiling a rare

\section{culprit}

\author{
Anamika Giri ${ }^{1}$, Sourya Acharya ${ }^{2}$, Amol Andhale ${ }^{3}$, Sunil \\ Kumar $^{4}$, Rohan Kumar Singh ${ }^{5}$, Abhijit Chandankhede ${ }^{6}$
}

\begin{abstract}
Neurofibromatosis type I (NF-I) is an autosomal dominant disorder. Epidemiologic data suggests it affects 1 in 3000 individuals. Various vascular abnormalities are reported to be associated with NF-1. Amongst these cerebrovascular anomalies are common in form of vascular stenosis or aneurysms. Arteriovenous malformations of the brain are still rarely found. We present a case of a 24 year old male who presented to this hospital with one episode of generalised tonic clonic seizure. After clinical examination and investigations the diagnosis of intracerebral haemorrhage due to rupture arteriovenous malformation and NF-1 was made. This report highlights the rare association of intracerebral vasculopthy with NF-1.
\end{abstract}

Keywords: Pectus Excavatum, Neurofibromatosis, Aneurysm

\section{INTRODUCTION}

Neurofibromatosis is a set of hereditary illnesses characterised by the formation of tumours on nerve tissue. Tumors are usually present in the neurological system; include the brain, spinal cord, and nerves. Neurofibromatosis is categorized into three types: neurofibromatosis 1 (NF1), neurofibromatosis 2 (NF2), and schwannomatosis. NF1 is frequently discovered in childhood, whereas NF2 and schwannomatosis are discovered in early adulthood (Oderich et al., 2007). The tumours in these illnesses are normally benign, but they can become cancerous at times. The signs and symptoms are usually not very severe. Hearing loss, learning disabilities, heart and cardiovascular complications, vision loss, and extreme pain are all possible effects of neurofibromatosis. Vascular abnormalities are quite common in NF-1 and seen in the renal, coronary and gastrointestinal vessels. There has been literature on vascular abnormalities in cerebral vessels in the form of occlusions, aneurysms, arteriovenous malformations and Av Fistulae.

\section{DISCOVERY} SCIENTIFIC SOCIETY

Copyright (c) 2022 Discovery Scientific Society. 


\section{CASE REPORT}

A 24 year old male patient came to the casualty with chief complaints of one episode of generalised tonic clonic seizures. This episode was associated with up rolling of eyes, tongue bite and frothing at mouth. Patient had no history of similar complaints in the past. There was no history of loss of consciousness. Patient had one episode of vomiting along with this episode. On examination; Patient was conscious but confused GCS 13/15. Pulse was 104/ minute, regular, Blood pressure was 130/80 mmHg. Neurologic examination revealed terminal neck stiffness. There were no cranial nerve abnormalities; power in all four limbs was $5 / 5$ as per MRC grade. There were no sensory deficits. Plantars were bilateral extensor. Multiple neurofibromas were palpable over body; (Figure-1) café-au-lait spots were present. Pectus excavatum deformity was seen on chest (Figure 2). Patient was started on injection Levitiracetam $500 \mathrm{mg}$ IV twice a day. In view of his complaints an emergency CT brain Plain was done which revealed haemorrhage in dependent part of fourth ventricle, third ventricle, left thalamus, perimesencephalic cistern with moderate dilatation of bilateral lateral ventricle (Figures 3, 4, 5 and 6). MRA revealed focal arteriovenous malformation along the left perimesencephalic cistern with arterial feeder from left posterior cerebral artery and venous drainage in internal cerebral veins (Figure 7). Patient was started on tablet Levetiracetam $500 \mathrm{mg}$ twice a day. Patient was referred to Neurosurgery for further management. The patient underwent flow directed selective onyx embolization of the feeding vessels and nidus. Post embolizations there were no complications. Currently after 2 months of discharge patient has no active complaints and there is no neurodeficit.

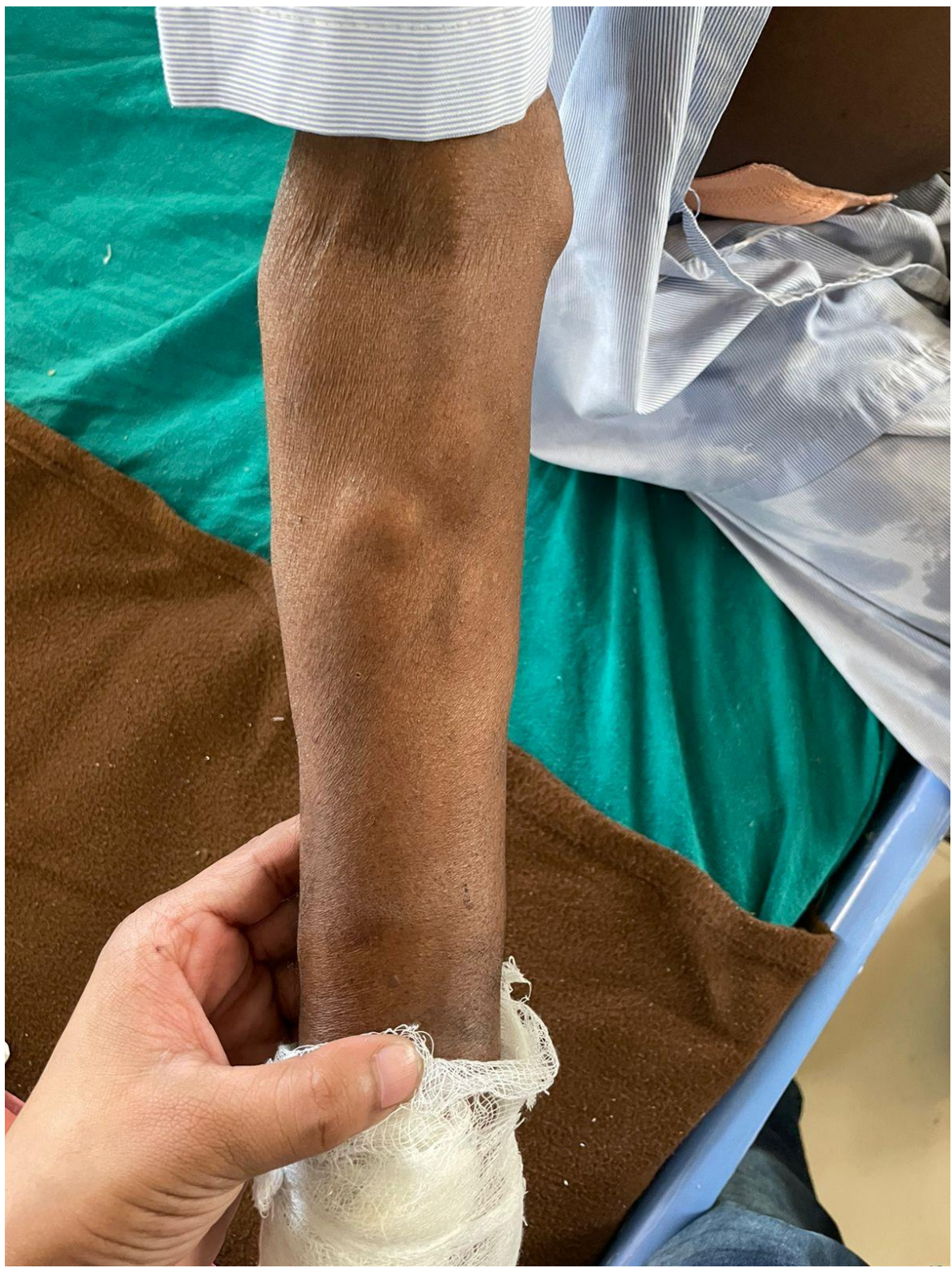

Figure 1 Showing multiple neurofibromas in forearm 


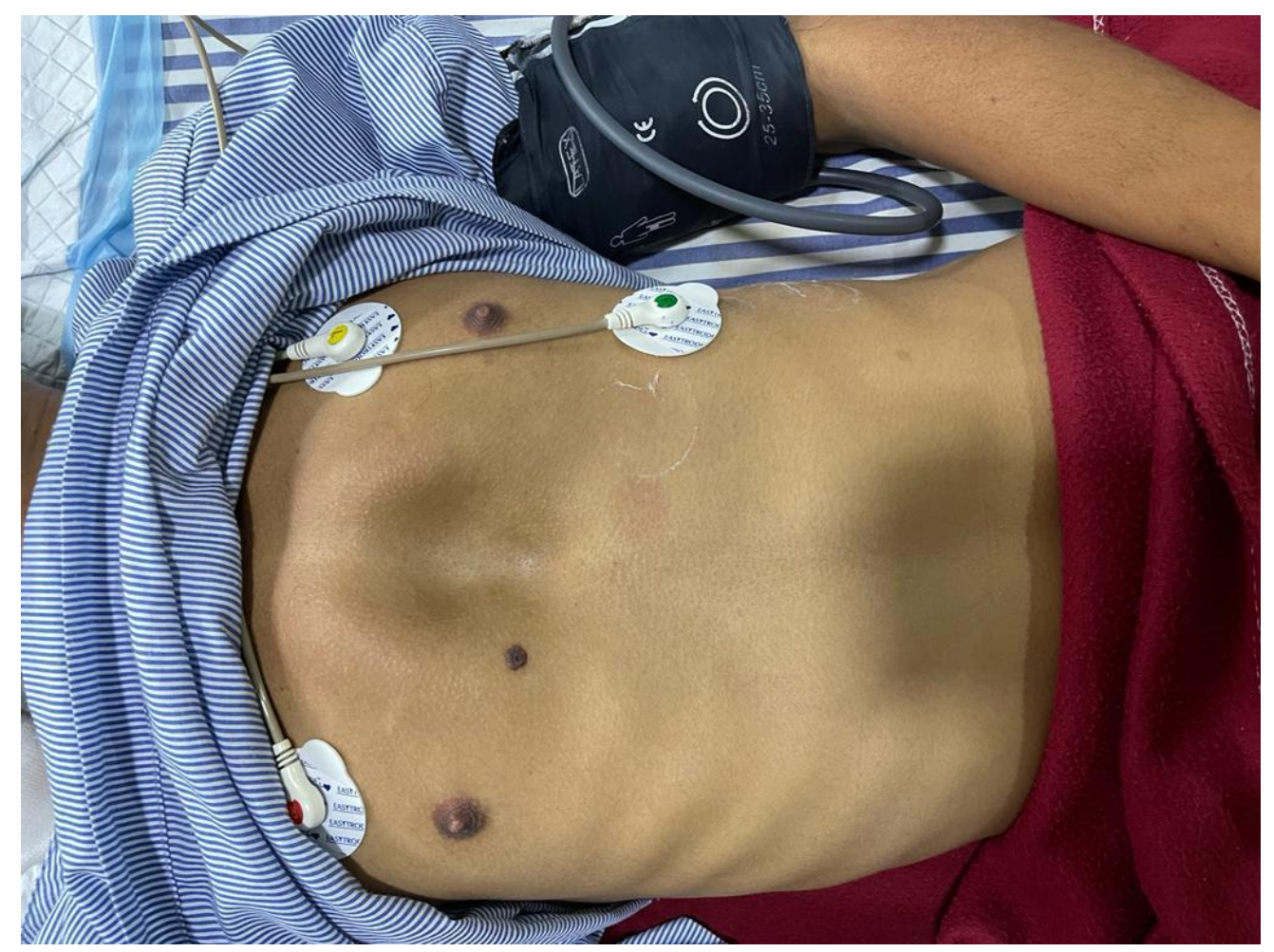

Figure 2 Showing PectusExcavatum

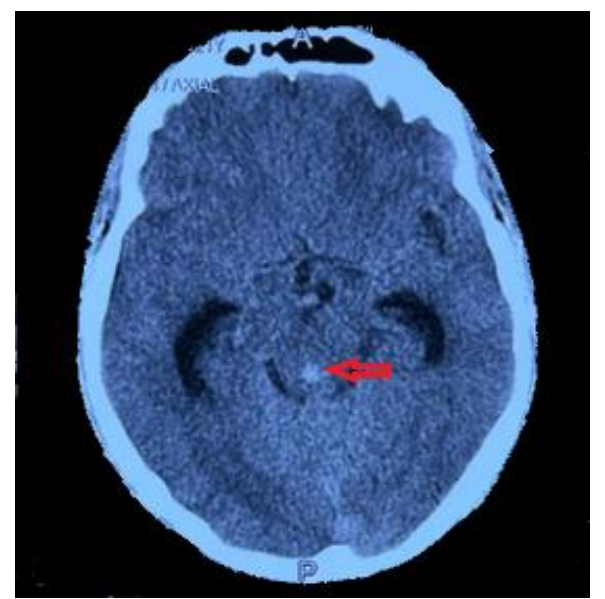

Figure $3 \mathrm{CT}$ axial section showing haemorrhage in dependant part of fourth ventricle.

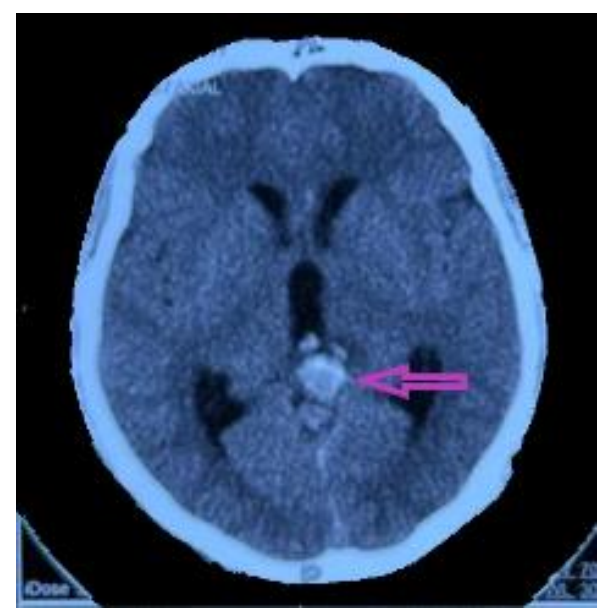

Figure $4 \mathrm{CT}$ axial section showing haemorrhage in the third ventricle 


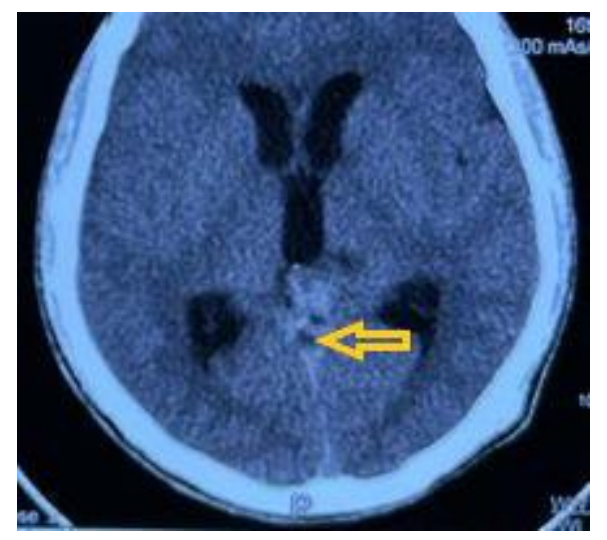

Figure 5 CT axial section showing ill definedhyperdensity in left thalamus and perimesencephalic cistern (bleed).

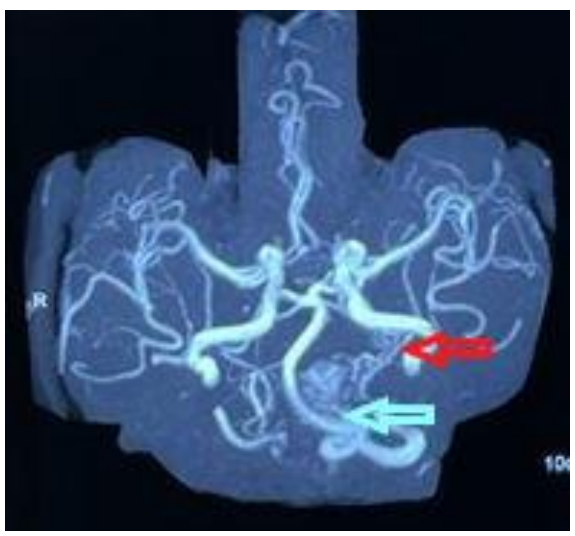

Figure 6 CT axial section CT axial section showing serpentine hypo to hyperdense lesion in left perimesencephalic cistern with moderate dilatation of bilateral lateral ventricle.

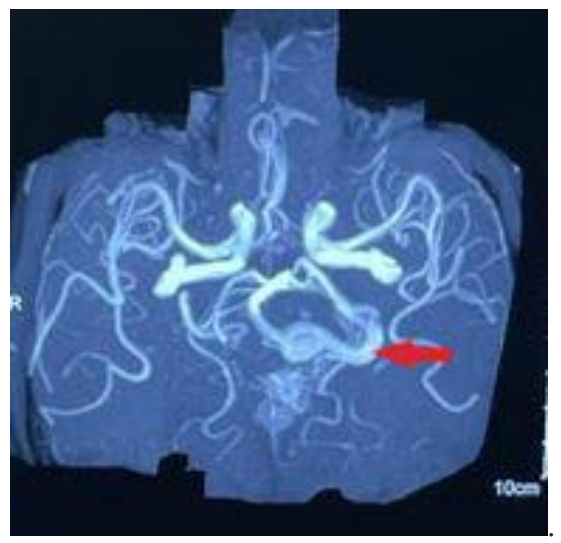

Figure 7 MRA showing focal arteriovenous malformation along the left perimesencephalic cistern with arterial feeder from left posterior cerebral artery and venous drainage in internal cerebral veins

\section{DISCUSSION}

Neurofibromatosis is usually a benign condition but it can sometimes have potentially life threatening squeale. One such sequalae is vasculopathy especially in the nervous system (Abbas et al., 2017; Singh et al., 2021). These vasculopathies range from stenosis, aneurysms, malformations, ruptures to even fistula formation. This doesn't usually affect the small vessels, medium to large sized vessels are usually affected. The exact cause of these vasculopathies is not known. It is said to be related to the activity of neurofibromin, the NF-1 gene's protein product (Evans, 2018). Neurofibromin has been demonstrated to limit cell proliferation by negatively regulating the RAS signalling pathway and positively controlling intracellular cAMP levels. The loss of gene neurofibromin expression in NF-1 enhances vascular smooth muscle cell proliferation via the RAS signalling system, resulting in proliferation of intima and arterial stenosis. It has also been discovered that neurofibromin is distributed differently in the smooth 
muscle layers and endothelium of different arteries, which may reveal why cerebral and renal vessels are more typically damaged by vascular dysplasia in NF-1 than the aorta.

A patient with neurofibromatosis may develop an AV fistula through one of two ways. Aneurysm development, leakage, and eventually rupture could be caused by dysplastic smooth muscle or neurofibromatous proliferation in the artery wall. Surgical excision, embolization or radiosurgery, (or mixes of these, in some situations) are all possibilities for treating AV malformations (Uhlmann and Plotkin, 2012). Approaches including one or another or combination of are both possible, information on outcomes is few, and data on outcomes is primarily based on guesswork from a set of cases participation of a multidisciplinary team (Roos, 1992).

It is recommended to apply a multidisciplinary approach which includes neurosurgery, radiation therapy and endovascular intervention, and make available all therapeutic options available. The purpose of treatment is to completely obliterate the arteriovenous malformation (Lin et al., 2000). Because partial obliteration appears to provide little or no benefit protection against haemorrhage, and may even increase the danger.

\section{CONCLUSION}

More research and reporting of such unique cases is the need of the hour. It will ensure prompt initiation of treatment and will significantly reduce mortality and morbidity. We aim to educate fellow physicians in ruling out such rare causes in a case of seizures by thorough examination and looking for conditions like neurofibromatosis and their sequalae which will be live saving and prevent any further complications.

Acknowledgement: We thank all the contributors involved in the study

\section{Author Contributions}

AG, SA, AA- initiated the idea of publication and contributed the intellectual content for the development of the manuscript RKS diagnosed the intraparenchymal bleed on CT and provided precise images of CT and MRA

SA, SK, AC reviewed, proof read and edited the manuscript

\section{Informed Consent}

Written \& Oral informed consent was obtained from all individual participants included in the study.

\section{Funding}

The study did not receive any external funding.

\section{Conflict of interests}

The authors declare that there are no conflicts of interests.

Data and materials availability

All data associated with this study are present in the paper.

\section{REFERENCES AND NOTES}

1. Abbas Z, Khani S, Zare J. Arteriovenous Malformation Underlying a Plexiform Neurofibroma: An Unusual Presentation. Indian Dermatol Online J 2017; 8(2):128-130.

2. Evans DG. Neurofibromatosis 2. Scott-Brown's Otorhinolaryngology Head and Neck Surgery. 2018; 126773.

3. Lin AE, Birch PH, Korf BR, Tenconi R, Niimura M, Poyhonen M, ArmfieldUhas K, Sigorini M, Virdis R, Romano C, Bonioli E. Cardiovascular malformations and other cardiovascular abnormalities in neurofibromatosis 1 . Am J Med Genet 2000; 95(2):108-17.
4. Oderich GS, Sullivan TM, Bower TC, Gloviczki P, Miller DV, Babovic-Vuksanovic D, Macedo TA, Stanson A. Vascular abnormalities in patients with neurofibromatosis syndrome type I: clinical spectrum, management, and results. JVS 2007; 46(3):475-484. doi: 10.1016/j.jvs.2007

5. Roos KL. The neurofibromatoses. ENTJ 1992; 71(10):512-9.

6. Singh A, Agrawal A, Vagha S. Plexiform neurofibroma: An Arduous entity. Medical Science 2021;25(111):1084-1087

7. Uhlmann EJ, Plotkin SR. Neurofibromatoses. Neurodegenerative diseases 2012; 266-77. 